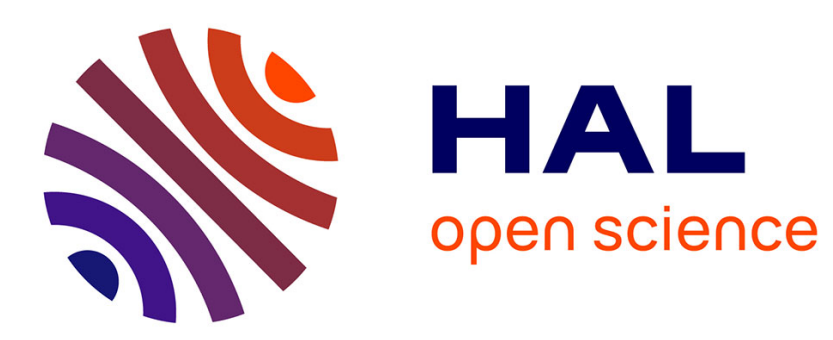

\title{
Modification of the Coulomb Logarithm due to Electron-Neutral Collisions
}

\author{
G J M Hagelaar, Z. Donko, N. Dyatko
}

\section{To cite this version:}

G J M Hagelaar, Z. Donko, N. Dyatko. Modification of the Coulomb Logarithm due to ElectronNeutral Collisions. Physical Review Letters, 2019, 123 (2), 10.1103/physrevlett.123.025004 . hal02327386

\section{HAL Id: hal-02327386 \\ https://hal.science/hal-02327386}

Submitted on 22 Oct 2019

HAL is a multi-disciplinary open access archive for the deposit and dissemination of scientific research documents, whether they are published or not. The documents may come from teaching and research institutions in France or abroad, or from public or private research centers.
L'archive ouverte pluridisciplinaire HAL, est destinée au dépôt et à la diffusion de documents scientifiques de niveau recherche, publiés ou non, émanant des établissements d'enseignement et de recherche français ou étrangers, des laboratoires publics ou privés. 


\title{
Modification of the Coulomb Logarithm due to Electron-Neutral Collisions
}

\author{
G. J. M. Hagelaar \\ LAPLACE, University of Toulouse, CNRS, 118 Route de Narbonne, 31062 Toulouse, France \\ Z. Donko \\ Institute for Solid State Physics and Optics, Wigner Research Centre for Physics, Hungarian Academy of Sciences, \\ P.O. Box 49, H-1525 Budapest, Hungary \\ N. Dyatko \\ State Research Center of Russian Federation Troitsk Institute for Innovation and Fusion Research, \\ ul. Pushkovykh 12, Troitsk, Moscow, Russia 108840
}

(Received 8 April 2019; published 10 July 2019)

\begin{abstract}
We demonstrate that in partially ionized plasmas, Coulomb scattering can be significantly perturbed by electron collisions with neutral gas particles, and that this effect can be incorporated in the Coulomb collision terms of the Boltzmann equation by a modification of the classical Coulomb logarithm. We show that Boltzmann transport calculations using this modified Coulomb logarithm are in excellent agreement, for a sensitive model problem and a wide range of conditions, with particle simulations describing the many-body Coulomb interactions from first principles.
\end{abstract}

DOI: 10.1103/PhysRevLett.123.025004

Coulomb scattering is an essential ingredient of charged particle transport phenomena in various systems such as gas discharges, fusion plasmas, space plasmas, or semiconductors [1,2]. The description of this scattering in terms of binary Coulomb collisions relies on the fact that it becomes ineffective at large interaction distances due to collective (many-body) screening effects. Traditionally this screening is modeled by applying a cutoff to the Rutherford scattering cross section [3],

$$
I=b d b /(d \cos \Theta)=\rho^{2} /(1-\cos \Theta)^{2},
$$

obtained from the classical relation between the scattering angle $\Theta$ and the impact parameter $b$ :

$$
1-\cos \Theta=2 /\left(1+b^{2} / \rho^{2}\right),
$$

where $\rho=\left|q_{1} q_{2}\right| /\left(4 \pi \epsilon_{0} \mu v^{2}\right)$ is a characteristic length corresponding to twice the distance of closest approach, determined by the vacuum permittivity $\epsilon_{0}$ and the charges $q$, reduced mass $\mu$, and relative velocity $v$ of the two interacting particles. The cutoff is applied for the impact parameter $b$ : it is assumed that interactions with $b>b_{\max }$, corresponding to $\Theta<\Theta_{\min }$, have no effect. This makes it possible to obtain nondivergent transport cross sections that account for the effects of Coulomb collisions on a mesoscopic level, averaged over all impact parameters, such as the momentum transfer cross section,

$$
\sigma_{m}=2 \pi \int_{\Theta_{\min }}^{\pi}(1-\cos \Theta) I \sin \Theta d \Theta=4 \pi \rho^{2} \ln \Lambda,
$$

where the cutoff distance appears in the Coulomb logarithm:

$$
\ln \Lambda=\ln \sqrt{1+\left(b_{\max } / \rho\right)^{2}} \approx \ln \left(b_{\max } / \rho\right) .
$$

This quantity arises as a prefactor in all Coulomb transport cross sections and in the Coulomb collision terms of the Boltzmann (Fokker-Planck) equation [4,5]. After some initial controversy about the value of $b_{\max }$, it has been well established since the 1950s [6] that for classical plasmas $b_{\max }$ is of the order of the Debye length $\lambda_{D}=$ $\left(\epsilon_{0} k T / e^{2} n_{e}\right)^{1 / 2}$, determined by the electron density $n_{e}$ and temperature $T$, and it is appropriate to evaluate $\ln \Lambda$ from average particle velocities $\left\langle v^{2}\right\rangle=3 k T / \mu$ as

$$
\ln \Lambda_{D}=\ln \left\langle b_{\max } / \rho\right\rangle=\ln \frac{12 \pi\left(\epsilon_{0} k T\right)^{3 / 2}}{e^{3} n_{e}^{1 / 2}},
$$

where we assumed singly charged particles $(q= \pm e)$ and added the subscript $D$ to refer to the Debye length. This classical expression holds for $\Lambda_{D} \gg 1$ and $k T<10 \mathrm{eV}$; at higher temperatures a quantum-mechanical correction is needed (due to $\rho \lesssim$ the de Broglie wavelength). Additional corrections exist for rapidly varying external fields or electron cyclotron oscillations faster than the plasma frequency, as discussed in Ref. [1].

In this Letter, we demonstrate that the above Coulomb logarithm must be modified when the Coulomb collisions occur simultaneously with collisions with neutral gas particles, in particular for electrons in weakly ionized plasmas in gas discharges. We start by presenting evidence 


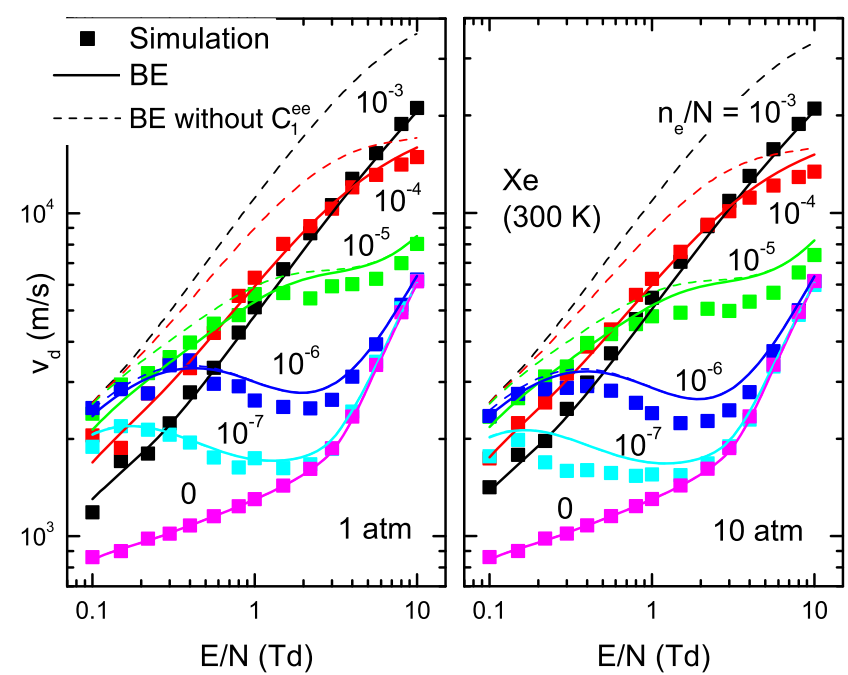

FIG. 1. Electron drift velocity in xenon as a function of reduced electric field strength $E / N$ (units Td $=10^{-21} \mathrm{~V} \mathrm{~m}^{2}$ ) for different ionization degrees $n_{e} / N$ (colors) and different gas pressures (leftand right-hand panels), calculated by different methods: firstprinciples particle simulation (symbols), Boltzmann equation (BE) with full electron-electron collision terms (solid lines), and Boltzmann equation with isotropic electron-electron collision term only (dashed lines).

from numerical simulations. We consider a basic dc discharge system, featuring electrons in a background of xenon gas of particle number density $N$ and subjected to a constant electric field of strength $E$ in an arrangement typical of swarm experiments [7], such that boundary effects are negligible. The electron distribution function and drift velocity in this system result from a subtle interplay between electric acceleration and momentum and energy exchanges in electron-neutral collisions and electron-electron Coulomb collisions, controlled mainly by the reduced field $E / N$ and the ionization degree $n_{e} / N$. For sufficiently low $E / N$, the drift velocity in xenon is very sensitive to $n_{e} / N$ and decreases with increasing $E / N$, a trend known as "negative differential conductivity," due to electron-electron collisions [8,9]. This constitutes an excellent test case for Coulomb collision theory, which we will use as our model problem.

The electron distribution function and drift velocity in the above system are traditionally calculated by solving the Boltzmann equation with collision terms for electron-neutral collisions and electron-electron Coulomb collisions, the latter involving the Coulomb logarithm. We will now check such Boltzmann calculations against results from full particle simulations describing the Coulomb interactions from first principles, without invoking binary Coulomb collisions. Figure 1 shows a comparison of the calculated electron drift velocity in xenon over a wide range of conditions.

Before discussing these results, we give some more information about the calculation methods used. The Boltzmann calculations were performed with the freeware

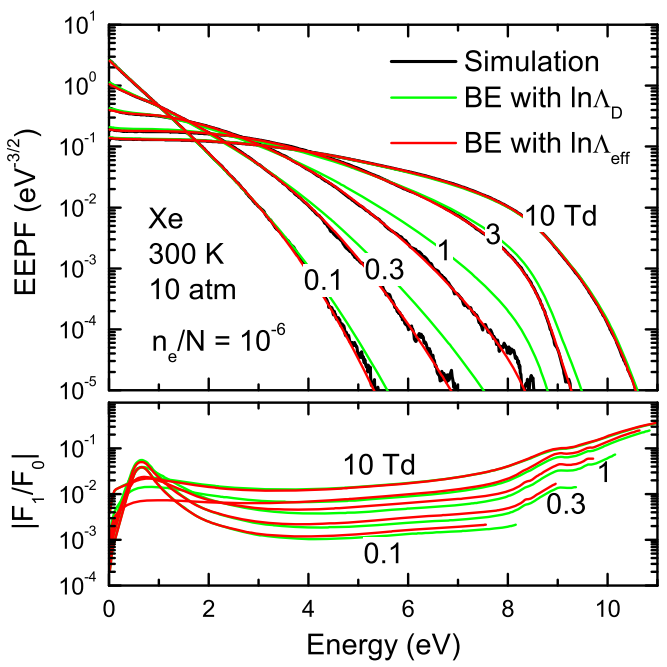

FIG. 2. Electron energy probability function (top) and anisotropy $\left|F_{1} / F_{0}\right|$ (bottom) in xenon with $n_{e} / N=10^{-6}$, for different $E / N$, obtained from first-principles particle simulations (black curves) and from the Boltzmann equation with classical (green curves) and modified (red curves) Coulomb logarithm.

Boltzmann equation solver BOLSIG+ $[10,11]$ based on the so-called two-term approximation, assuming that the electron (phase space) distribution function can be cast in the following functional form:

$$
f=\frac{n_{e}}{2 \pi \gamma^{3}}\left[F_{0}(\varepsilon)+F_{1}(\varepsilon) \cos \chi\right]
$$

where $F_{0}$ and $F_{1}$ are functions of the electron energy $\varepsilon$ only, $\chi$ is the electron velocity angle with respect to the direction of the electric field, and $\gamma=\left(2 / m_{e}\right)^{1 / 2}$ is a constant with $m_{e}$ the electron mass. The function $F_{0}$ corresponds to the electron energy probability function (EEPF), generally non-Maxwellian in gas discharge plasmas, while $F_{1}$ represents the anisotropy of the distribution function due to the electric field, resulting in the electron drift velocity $v_{d}=(\gamma / 3) \int_{0}^{\infty} \epsilon F_{1} d \epsilon$, where $F_{1} \ll F_{0}$ (see Fig. 2). In this two-term approximation, the Boltzmann equation takes the form of two coupled integro-differential equations for $F_{0}$ and $F_{1}$ :

$$
\begin{gathered}
-\frac{\gamma e E}{3 \varepsilon^{1 / 2}} \frac{\partial F_{1}}{\partial \varepsilon}=C_{0}^{\mathrm{en}}\left[F_{0}\right]+C_{0}^{\mathrm{ee}}\left[F_{0}\right], \\
-\gamma e E \varepsilon^{1 / 2} \frac{\partial F_{0}}{\partial \varepsilon}=-N \gamma \varepsilon^{1 / 2} \sigma_{m}^{\mathrm{en}} F_{1}+C_{1}^{\mathrm{ee}}\left[F_{0}, F_{1}\right],
\end{gathered}
$$

where $C_{0}^{\text {en }}$ describes electron energy losses due to different kinds of elastic and inelastic collisions with neutral gas particles, $\sigma_{m}^{\text {en }}$ is an effective cross section for the total momentum transfer in these collisions, and $C_{0}^{\mathrm{ee}}$ and $C_{1}^{\mathrm{ee}}$ describe the effects of electron-electron Coulomb collisions on $F_{0}$ and $F_{1}$. For a detailed description of these collision 
terms and their respective effects on the electron distribution function, we refer to Ref. [11]. The Coulomb collision terms are proportional to the electron density and the Coulomb logarithm:

$$
C_{0,1}^{\mathrm{ee}} \propto n_{e} \ln \Lambda_{D},
$$

where $\ln \Lambda_{D}$ is calculated using $k T=(2 / 3) \int_{0}^{\infty} \varepsilon^{3 / 2} F_{0} d \varepsilon$ and is in the range 5-10. The $C_{1}^{\text {ee }}$ term plays an important role in the Spitzer conductivity for fully ionized plasmas [12] but is generally neglected in the gas discharge literature, following Ref. [13]; for this reason we also included in Fig. 1 results obtained without this term. These Boltzmann calculations were done with high numerical resolution (400 energy points) and strict convergence criteria $\left(<10^{-8}\right.$ fractional energy balance residue), yet the computation time was negligible ( $<1 \mathrm{~s}$ per solution).

The numerical code used for the particle simulations was previously presented in Ref. [14] and used in Ref. [9]; it is based on a combination of Monte Carlo techniques for electron-neutral collisions and molecular dynamics techniques to describe the electron-electron Coulomb interactions from first principles, explicitly taking into account the long-range Coulomb forces between the particles. Very computation intensive, these simulations serve primarily as approximation-free benchmarks for Boltzmann calculations. Because of the extremely small simulation time step $\left(\approx 6 \times 10^{-17}\right.$ s) required for a stable and accurate integration of electron trajectories at their closest approach, a run time of about 1 month (on a single CPU) was necessary for each data point in Fig. 1, using 500 particles. We extensively verified that all results are properly converged and free from numerical artifacts (apart from the inevitable statistical noise). The same electron-neutral cross section data $[15,16]$ were used in all calculations.

The comparison in Fig. 1 shows that the Boltzmann calculations are in excellent agreement with the particle simulations when $n_{e} / N$ is either zero (no Coulomb scattering) or relatively high $\left(\geq 10^{-3}\right)$, provided that the $C_{1}^{\text {ee }}$ term is included in the Boltzmann calculations (solid lines). This is strong evidence that both codes are free from serious implementation errors and that the approximations involved in the Boltzmann calculations are valid here. However, at intermediate ionization degrees, the drift velocities predicted by the Boltzmann calculations are systematically higher than those measured in the particle simulations. Also, the electron energy distribution functions deviate considerably, as is illustrated by Fig. 2 (black vs green curves) for $n_{e} / N=10^{-6}$. These discrepancies become more important with increasing gas pressure, which suggests that they are due to shortcomings of the Coulomb logarithm, because this is the only element in the Boltzmann calculations that is affected by the gas density, via $n_{e}=N\left(n_{e} / N\right)$, given the same $n_{e} / N$ and $E / N$.

We will now demonstrate that these discrepancies are indeed due to the Coulomb logarithm, via the following mechanism: the electron-neutral collisions are so frequent that they perturb the electron-electron interactions and reduce their effects, which translates into a decrease of $\ln \Lambda$. To see qualitatively how this works, consider a Coulomb collision between two electrons with impact parameter $b$, causing their relative velocity to be scattered over an angle $\Theta$. This takes a certain time to happen, which is longer for larger $b$. If during that time one of the electrons is scattered isotropically by colliding with a neutral particle, this breaks the Coulomb collision up into two parts with partial scattering angles in different directions, independent from each other. This reduces the total exchange of momentum and energy between the two electrons: since the exchange is proportional to $(1-\cos \Theta) \approx \Theta^{2} / 2$ for each independent angle $\Theta$, the total exchange for two independent half-angles, $2 \times(\Theta / 2)^{2} / 2=\Theta^{2} / 4$, is twice as small. Extrapolating this argument, we expect that if there occur $m$ electron-neutral collisions during a Coulomb collision, then the energy exchange of that Coulomb collision is reduced by a factor $1 /(m+1)$. And because $m$ tends to increase as a function of $b$, Coulomb collisions with large impact parameters become ineffective.

Let us work this out more precisely. From a straightforward classical orbit calculation one finds that, during an unperturbed Coulomb collision, the angle $\theta$ of the relative electron velocity changes in time $t$ according to

$$
\frac{t}{\tau}=\frac{b \tan \theta}{\sqrt{\rho^{2}-b^{2} \tan ^{2} \theta}}+\frac{\rho}{2 \sqrt{b^{2}+\rho^{2}}} \ln \frac{\rho+b \tan \theta}{\rho-b \tan \theta},
$$

where $\tau=\sqrt{b^{2}+\rho^{2}} / v$ and we took $t=0$ and $\theta=0$ at closest approach. An approximate solution of Eq. (10) can be found by neglecting the second term on the right-hand side:

$$
\theta(t) \approx \arctan \frac{\rho t}{b \sqrt{t^{2}+\tau^{2}}}
$$

From this equation we see immediately that, while in total the velocity is scattered by an angle $\Theta=\theta(\infty)-\theta(-\infty)$ as in Eq. (2), most of the scattering happens near the point of closest approach, over a time of the order of a few $\tau$. Hence we assume that the average number of electron-neutral collisions that occur during a Coulomb collision can be written as

$$
m=\alpha \nu \tau=\frac{\alpha \nu}{v} \sqrt{b^{2}+\rho^{2}},
$$

where $\nu$ is the electron-neutral collision frequency and $\alpha$ is a numerical constant of the order of a few times unity. We also assume that these $m$ collisions split the angle $\Theta$ equally into $m+1$ uncorrelated angles $\Theta /(m+1)$. Note that both $m$ and $\Theta$ depend on the impact parameter $b$, which jumps to a different value at each electron-neutral collision, so these 
$m$ electron-neutral collisions do not actually occur one after another during the same Coulomb collision, but rather within different Coulomb collisions whose momentary orbits correspond to the same $b$. On a mesoscopic level though, when integrating over all possible impact parameters, this does not matter: all that counts is that 1 scattering event of angle $\Theta$ gets effectively replaced by $m+1$ scattering events of angle $\Theta /(m+1)$. Applying this replacement to the momentum transfer cross section Eq. (3), we find

$$
\begin{aligned}
\sigma_{m}^{\text {eff }} & =2 \pi \int_{\Theta_{\min }}^{\pi}(m+1)\left(1-\cos \frac{\Theta}{m+1}\right) \sin \Theta d \Theta \\
& \approx 2 \pi \int_{0}^{b_{\max }} \frac{1-\cos \Theta}{m+1} b d b=4 \pi \rho^{2} \ln \frac{\Lambda+M}{1+M},
\end{aligned}
$$

where $M=(\alpha \nu / v) \sqrt{b_{\max }^{2}+\rho^{2}}$. This momentum transfer cross section differs from that in Eq. (3) only by the Coulomb logarithm, which now contains an additional parameter $M$ due to electron-neutral collisions.

Clearly the assumption that $\Theta$ is broken up into equal parts is a crude approximation; the true breakup is more or less unequal due to the varying rate of change of $\theta(t)$ and the random occurrence of the electron-neutral collisions. A more rigorous version of the above calculation, taking into account the full evolution of $\theta(t)$ from Eq. (10) and the randomness of the electron-neutral collisions, is represented by the following integral:

$$
\begin{aligned}
\sigma_{m}^{\mathrm{eff}}= & 2 \pi \int_{0}^{b_{\max }} b d b \int_{-\infty}^{\infty} 2 \nu d t_{1} \int_{t_{1}}^{\infty} d t_{2} \\
& \times 2 \nu e^{-2 \nu\left(t_{2}-t_{1}\right)}\left[1-\cos \left(\theta_{2}-\theta_{1}\right)\right] .
\end{aligned}
$$

Here the subscripts 1 and 2 refer to two consecutive electron-neutral collisions, marking the beginning and ending of an independent portion of a Coulomb collision, $2 \nu d t_{1}$ is the probability that such a portion starts between $t_{1}$ and $t_{1}+d t_{1}$ (because one of the two electrons collides with a neutral), and $2 \nu e^{-2 \nu\left(t_{2}-t_{1}\right)} d t_{2}$ is the probability that it then ends between $t_{2}$ and $t_{2}+d t_{2}$. This integral cannot be calculated analytically but is readily evaluated numerically, changing the integration variables $t \rightarrow \theta$ to enable substitution of Eq. (10). Moreover, in the limit of many electron-neutral collisions, $\nu \rho \gg v$, Eq. (14) yields

$$
\sigma_{m}^{\mathrm{eff}} \approx 4 \pi \rho^{2} \frac{v}{3 \nu \rho} .
$$

This can be used to calibrate the constant $\alpha$ in the parameter $M$ of the approximate expression [Eq. (13)]: in order to reproduce the limit Eq. (15), one must set $\alpha=3$. Figure 3 shows a comparison between the effective Coulomb logarithm $\ln \Lambda_{\text {eff }}=\sigma_{m}^{\text {eff }} /\left(4 \pi \rho^{2}\right)$ calculated numerically from Eq. (14) and the approximate Eq. (13) with $\alpha=3$, over a large range of conditions. It turns out that the

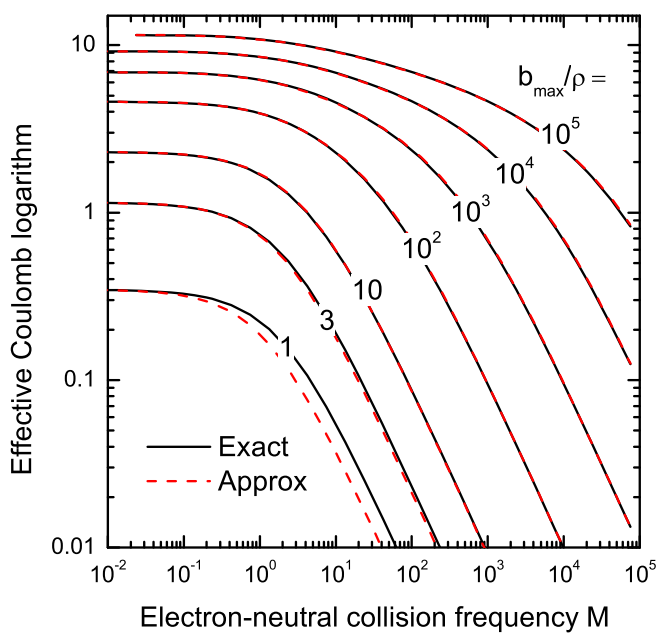

FIG. 3. Comparison between the effective Coulomb logarithm $\sigma_{m}^{\text {eff }} /\left(4 \pi \rho^{2}\right)$ from Eq. (14) and from the approximate Eq. (13) with $\alpha=3$.

agreement is remarkably good, better than $1 \%$, except at very low values of $\Lambda$ when the binary collision theory is no longer valid anyway. We therefore conclude that it is safe to use Eq. (13) for all conditions of interest.

Finally, averaging over the electron energy distribution and substituting $b_{\max }=\lambda_{D}$, we obtain the following convenient expression for the effective Coulomb logarithm in the presence of electron-neutral scattering:

$$
\ln \Lambda_{\mathrm{eff}}=\ln \frac{\Lambda_{D}+M}{1+M}, \quad M \approx \frac{\sqrt{6}}{2} \frac{\langle\nu\rangle}{\omega_{p}},
$$

where $M$ is evaluated from the ratio of the mean electronneutral collision frequency $\langle\nu\rangle$ to the plasma frequency
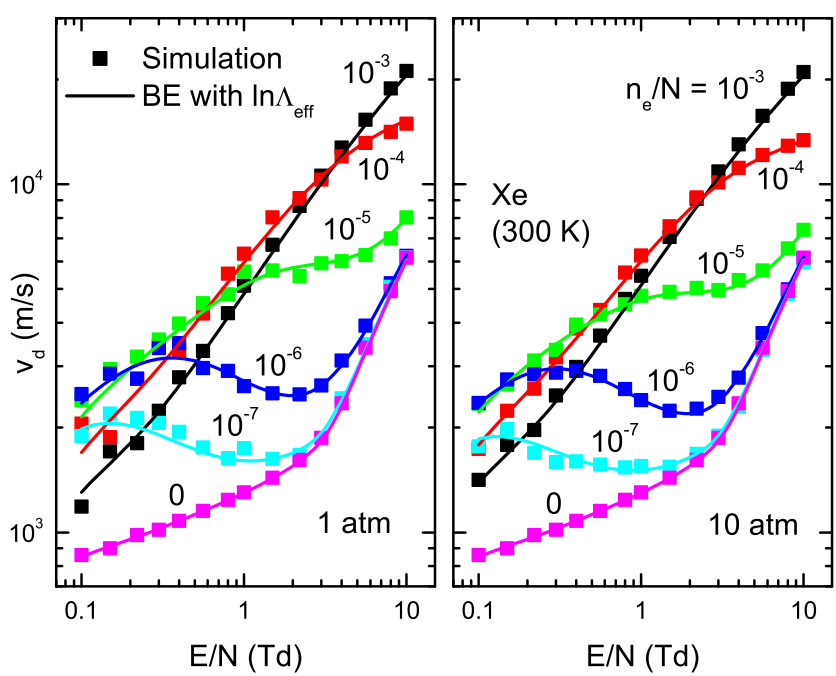

FIG. 4. Same as Fig. 1 but using the modified Coulomb logarithm of Eq. (16) in the Boltzmann calculations (solid lines), with full electron-electron collision terms. 
$\omega_{p}=\left(e^{2} n_{e} / \epsilon_{0} m_{e}\right)^{1 / 2}$, corresponding qualitatively also to the ratio of $\lambda_{D}$ to the electron-neutral mean free path.

We implemented this effective Coulomb logarithm into the Boltzmann calculations of Fig. 1, with $\langle\nu\rangle=\gamma N \int_{0}^{\infty}$ $\varepsilon \sigma_{m}^{\mathrm{en}} F_{0} d \varepsilon$, and found that all results are now in excellent agreement with those from the first-principles particle simulations, to within statistical uncertainties over the full range of conditions, which confirms the validity of Eq. (16). This holds not only for the electron drift velocity, shown in Fig. 4, but for the entire electron distribution function, illustrated by Fig. 2 (black and red curves). For completeness, we note that electron transport in quasineutral plasmas at high $n_{e} / N\left(\gtrsim 10^{-5}\right)$ can be significantly affected by electron-ion momentum transfer [11], which was not included in these calculations, but is unrelated to the behavior of the Coulomb logarithm of interest here.

In summary, we introduced a modification to the classical Coulomb logarithm that takes into account the effect of electron-neutral collisions during Coulomb scattering events. The correctness of this modification was tested and confirmed for a sensitive electron transport problem, for which inconsistencies between the results from the Boltzmann equation and from first-principles particle simulations were shown to be resolved. In view of the general nature of the underlying physical mechanism, we expect this modification to hold in general for partially ionized classical plasmas and to be significant whenever the electron-neutral collision frequency exceeds the plasma frequency. Future work will have to explore the consequences of this modification for different plasma problems and scenarios.

One of the authors (Z.D.) acknowledges support from the Hungarian Office for Research, Development and Innovation, Grant No. NKFIH 119357.

*gerjan.hagelaar@laplace.univ-tlse.fr

[1] I. P. Shkarofsky, T. W. Johnston, and M. P. Bachynski, The Particle Kinetics of Plasmas (Addison-Wesley, Reading, MA, 1966).

[2] D. Chattopadhyay and H. J. Queisser, Electron scattering by ionized impurities in semiconductors, Rev. Mod. Phys. 53, 745 (1981).
[3] J. D. Jackson, Classical Electrodynamics, 3rd ed. (Wiley, New York, 1999).

[4] S. Chandrasekar, Stochastic problems in physics and astronomy, Rev. Mod. Phys. 15, 1 (1943).

[5] M. N. Rosenbluth, W. MacDonald, and D. L. Judd, FokkerPlanck equation for an inverse-square force, Phys. Rev. 107, 1 (1957).

[6] R. S. Cohen, L. Spitzer, and P. M. Routly, The electrical conductivity of an ionized gas, Phys. Rev. 80, 230 (1950).

[7] Z. L. Petrovic, S. Dujko, S. Maric, G. Malovic, Z. Nikitovic, O.

Sasic, O. Jovanovic, V. Stojanovic, and M. RadmilovicRadenovic, Measurement and interpretation of swarm parameters and their application in plasma modelling, J. Phys. D 42, 194002 (2009).

[8] N. L. Aleksandrov, N. A. Dyatko, I. V. Kochetov, A. P. Napartovich, and D. Lo, Negative differential conductivity of electrons in pure rare gases, Phys. Rev. E 53, 2730 (1996).

[9] Z. Donko and N. Dyatko, First-principles particle simulation and Boltzmann equation analysis of negative differential conductivity and transient negative mobility effects in xenon, Eur. Phys. J. D 70, 135 (2016).

[10] G. J. M. Hagelaar and L. C. Pitchford, Solving the Boltzmann equation to obtain electron transport coefficients and rate coefficients for fluid models, Plasma Sources Sci. Technol. 14, 722 (2005).

[11] G. J. M. Hagelaar, Coulomb collisions in the Boltzmann equation for electrons in low-temperature gas discharge plasmas, Plasma Sources Sci. Technol. 25, 015015 (2016).

[12] L. Spitzer and R. Harm, Transport phenomena in a completely ionized gas, Phys. Rev. 89, 977 (1953).

[13] S. D. Rockwood, Elastic and inelastic cross sections for electron-Hg scaterring from $\mathrm{Hg}$ transport data, Phys. Rev. A 8, 2348 (1973).

[14] Z. Donko, First principles calculation of the effect of Coulomb collisions in partially ionized gases, Phys. Plasmas 21, 043504 (2014).

[15] M. Hayashi, Bibliography of electron and photon cross sections with atoms and molecules published in the 20th century, Technical Report No. NIFS-DATA-079, NIFS, 2003.

[16] J. L. Pack, R. E. Voshall, and A. V. Phelps, Drift velocities of slow electrons in krypton, xenon, deuterium, carbon monoxide, carbon dioxide, water vapor, nitrous oxide, and ammonia, Phys. Rev. 127, 2084 (1962). 